\title{
Chemical synthesis and antibacterial activity of novel-shaped silver nanoparticles
}

\author{
Pham Van Dong ${ }^{1 *}$, Chu Hoang Ha², Le Tran Binh² and Jörn Kasbohm³
}

\begin{abstract}
Silver nanoparticles are useful for medical applications due to their strong antibacterial activity. The antibacterial activity can be tuned by controlling the size and shape of the prepared silver nanoparticles. In this work, silver nanoparticles with different sizes and shapes were synthesized by solution phase routes, and their interactions with Escherichia coli were studied. Triangular silver nanoprisms were prepared by the reduction of silver nitrate at room temperature in the presence of polyvinylpyrrolidone, sodium citrate, hydrogen peroxide and sodium borohydride. Spherical silver nanoparticles were also prepared using silver nitrate as metal precursor and sodium citrate as well as sodium borohydride as reducing agents. The morphologies and structures of the nanoparticles were characterized by transmission electron microscopy, UV-visible spectroscopy and X-ray diffraction. The results indicated that spherical silver nanoparticles were obtained with different average sizes of 4, 21 and $40 \mathrm{~nm}$, respectively. The edged silver nanoprisms containing mainly $\{111\}$ lattice planes were obtained in the range size of 25 to $400 \mathrm{~nm}$. The antibacterial study revealed that the edged triangular silver nanoprisms with $\{111\}$ lattice planes exhibited the strongest antibacterial property, compared with spherical nanoparticles. Our study demonstrated that triangular silver nanoprisms with sharp edges and sharp vertexes also display a good antibacterial activity in comparison to other shaped nanoparticles.
\end{abstract}

Keywords: silver nanoparticles, silver nanoprisms, antibacterial activity, TEM, UV-vis spectroscopy

\section{Background}

Silver nanoparticles (AgNPs) are subjected to new engineering technologies with extraordinarily novel resultant morphologies and characteristics. Silver is engineered into ultrasmall particles (normally between 1 to $100 \mathrm{~nm}$ ) [1]. At nanoscale, the ultrasmall particle size leads to ultralarge surface area per mass where a large number of atoms are in immediate contact with ambiance and readily available for reaction. Unique interactions with bacteria and virus have been demonstrated by silver nanoparticles of certain size ranges and shapes [2].

Recent publications have focused on the synthesis and antibacterial characterization of silver nanoparticles. $\mathrm{Nu}$ merous approaches have been used to prepare silver nanoparticles. Examples of physical technique to prepare AgNPs are photochemical synthesis [3], laser ablation [4], phase transfer processes [5], microemulsion [6],

\footnotetext{
*Correspondence: phm.vandong@gmail.com

1 Institute of Material Science, Vietnam Academy of Science and Technology, 18 Hoang Quoc Viet Street, CauGiay District, Hanoi 010000, Vietnam Full list of author information is available at the end of the article
}

microwave treatment [7] and $\gamma$-irraditation [8], which have been used for preparation in both solution and solid phases of silver nanoparticles. Biosynthetic [9-11] and physicochemical approaches [12] have been extensively used to prepare silver nanoparticles with the aim of improving the conventional methods. Although these nanofabrication techniques have been demonstrated to be an alternative to solution-solid phase methods, the chemical methods are more versatile. The chemical reduction methods are involved in the preparation of silver nanoparticles with well-controlled size in which silver ions are reduced by reductants and stabilizing or protecting agents to prevent these nanoparticles from agglomeration. A variety of chemical approaches have also been utilized to produce silver nanoparticles with different size distribution and different shapes [13-16].

Silver nanoparticles have received considerable attention as antimicrobial agents and have been shown to be an effective antimicrobial agent [17]. The major mechanism of the antibacterial properties of silver nanoparticles is by anchoring and penetrating of silver particles to the

\section{它}


bacterial cell wall; once inside the cell, silver nanoparticles modulate the cellular signaling by dephosphorylating putative key peptide substrates, which are critical for cell viability and division [18]. Antimicrobial capability of silver nanoparticles allows them to be suitably employed in applications and products such as water filter materials [19], textiles [20] and medical applications [21].

In the previous studies, many works have mainly focused on the synthesis and study of the antibacterial activity of silver nanoparticles in a general manner with respect to the antibacterial activity through the interaction of spherical silver nanoparticles with bacterial cell [22-24]. However, there were few studies investigating the antibacterial activity of silver nanoparticles that depends on the shapes and crystal structures of silver nanoparticles. The first comparative study on the bactericidal properties of silver particles of different shapes was conducted by Pal et al. [25]. In that work, differently morphological silver particles such as spherical, rodshaped and truncated triangular silver nanoparticles were used to treat microorganism. Interestingly, the truncated triangular nanoparticles displayed the strongest biocidal action, compared with the spherical and rod-shaped nanoparticles, due to their crystal structures whose $\{111\}$ lattice planes contain a large number of atoms. It has been demonstrated that the reactivity of silver is favored by high-atom-density facets. Thus, a high reactivity of the truncated triangular nanoplates, as found in that study, in comparison to other particles that contain fewer than $\{111\}$ facets, like spherical or rodshaped particles, is expected. However, a question should be raised whether only the truncated triangular nanoparticles mainly composed of $\{111\}$ lattice planes show the best antibacterial activity. Thus, we carried out a thorough study on the antibacterial activity of novel triangular nanoprisms in comparison to the previous study.

Our aims in the present contribution were to synthesize silver nanoparticles with spherical and triangular shapes by chemical route and to investigate the antibacterial activity of these nanostructures. We presented the preparation of triangular silver nanoprisms with very sharp edges and sharp vertexes using a roomtemperature route that allows the growth of nearly unimodal distributions so that the in-plane dipole plasmon resonance is shifted to wavelengths above $600 \mathrm{~nm}$. In this method, the hydrogen peroxide $\left(\mathrm{H}_{2} \mathrm{O}_{2}\right)$ plays as the oxidizing agent to induce the transformation into nanoprisms at room temperature. We also synthesized the spherical silver nanoparticles using silver nitrate as precursor and other common reducing and stabilizing agents for the investigation of size effect on the antibacterial properties of silver. The synergistic effect of these differently sized and shaped silver nanoparticles on a strain of Escherichia coli was qualitatively evaluated by zone inhibition method. The antibacterial activity of triangular nanoprisms was investigated with particular regard to the morphology and crystal structures.

\section{Methods \\ Materials}

Silver nitrate $\left(\mathrm{AgNO}_{3}, 99.99 \%\right)$, trisodium citrate dehydrate $\left(\mathrm{C}_{6} \mathrm{H}_{5} \mathrm{O}_{7} \mathrm{Na}_{3} .2 \mathrm{H}_{2} \mathrm{O}\right.$, 99.99\%), sodium borohydride $\left(\mathrm{NaBH}_{4}, 99.99 \%\right)$, hydrogen peroxide $\left(\mathrm{H}_{2} \mathrm{O}_{2}\right)$, polyvinylpyrrolidone (PVP; $\mathrm{Mw}=40,000)$ were purchased from Roth, Karlsruher Rheinhafen, Germany. E. coli DH5 $\alpha$ subjected to this analysis was from the Institute of Biotechnology, VAST, Vietnam. Deionized water was used throughout the experiment.

\section{Preparation of silver nanostructures}

To synthesize different-sized AgNPs, three methods were used. In the first method, the spherical AgNPs were prepared according to the literature procedure by Fang et al. [26], by reducing aqueous $\mathrm{AgNO}_{3}$ with sodium citrate at boiling temperature. In typical procedure, $50 \mathrm{ml}$ of $0.001 \mathrm{M} \mathrm{AgNO}_{3}$ was heated to boiling. To this solution, $5 \mathrm{ml}$ of $1 \%$ trisodium citrate was added drop by drop. The solution was heated at boiling point under continuous stirring. The reaction was allowed to take place until the color changed to a greenish yellow solution. The solution was then cooled to room temperature. The AgNPs in this solution were called citrate-AgNPs.

In the second method, spherical silver nanoparticles were prepared by reducing aqueous silver nitrate in the presence of $\mathrm{NaBH}_{4}$. Briefly, an aqueous solution of $\mathrm{NaBH}_{4}(0.002 \mathrm{M})$ was added to a flask. The solution was stirred and cooled for another $20 \mathrm{~min}$. Two milliliters of $0.001 \mathrm{M} \mathrm{AgNO}_{3}$ was dropped into the stirred $\mathrm{NaBH}_{4}$ solution at approximately 1 drop per second. Stirring the solution was stopped as soon as all of the $\mathrm{AgNO}_{3}$ was added. Particles produced by this method were called borohydride-AgNPs.

In the third method, AgNPs were synthesized by using $\mathrm{NaBH}_{4}$ and PVP as reducing and stabilizing agents, respectively. An aqueous solution of trisodium citrate $(0.5 \mathrm{ml}, 30 \mathrm{mM})$ was added into a flask $(50 \mathrm{ml}$ deionized water), and then, an aqueous solution of $\mathrm{AgNO}_{3}(1 \mathrm{ml}$, $5 \mathrm{mM}$ ) was added. Freshly prepared $\mathrm{NaBH}_{4}$ aqueous solution $(0.5 \mathrm{ml}, 50 \mathrm{mM})$ was quickly added, and the suspension immediately turned a light yellow color. After $30 \mathrm{~s}$, an aqueous solution of PVP $(0.5 \mathrm{ml}, 5 \mathrm{mg} / \mathrm{ml}$, $\mathrm{Mw}=40,000)$ was also added. The suspension changed to a darker yellow color after reaction had proceeded for another $30 \mathrm{~min}$. These silver particles were marked PVP-AgNPs.

Finally, triangular nanoprisms (triangular-AgNPs) were synthesized following the procedure conducted by 
Torres and coworkers [27]. PVP:AgNO 3 precursor solution was prepared initially, which consists of $\mathrm{AgNO}_{3}$ solution $(1 \mathrm{ml}, 5 \mathrm{mM})$ in $50 \mathrm{ml}$ deionized water in the presence of PVP ( $3 \mathrm{ml}, 32 \mathrm{mg}, \mathrm{Mw}=40,000)$ which acts as the capping agent. Then, sodium citrate $(3 \mathrm{ml}$, $30 \mathrm{mM}$ ) and hydrogen peroxide were added to the solution under constant stirring at $400 \mathrm{rpm}$. The reaction took place in the dark. The solution changed from faint yellow to a light blue after $30 \mathrm{~min}$. The reaction was allowed to continue for $5 \mathrm{~h}$.

The size and morphology of the nanoparticles were analyzed with a transmission electron microscope (TEM; JEOL 1210, JEOL Ltd., Tokyo, Japan), and the optical absorption features of Ag colloids in the UV-visible (UVvis) range of 200- to $800-\mathrm{nm}$ wavelength were measured using a spectrophotometer (SHIMADZU UV-1650PC, Columbia, MD, USA). The samples for X-ray diffraction (XRD) analysis were made by taking a small amount of solution from the bottle and drying it on a quartz plate.

\section{Test for antibacterial activity of Ag nanoparticles}

The antibacterial susceptibility of silver nanoparticles was evaluated using the zone inhibition method. A 100$\mu$ sample of bacterial suspension cultured in LB (with a concentration of $10^{5}$ to $10^{7} \mathrm{CFU} / \mathrm{ml}$ of $E$. coli) was spread on a nutrient agar plate. The plates were then holed and supplemented with spherical silver nanoparticles and triangular silver nanoprisms to determine the different antibacterial properties depending on size and shapes; the plates were then incubated further at $37{ }^{\circ} \mathrm{C}$. The zones of inhibition were calculated after $24 \mathrm{~h}$ of incubation.

\section{Results and discussion Morphology Spherical Silver Nanoparticles}

Figure 1a,b shows TEM micrographs with different magnifications of citrate-AgNPs, which were prepared by citrate reduction. The particles are in a broad size distribution from 15 to $60 \mathrm{~nm}$ with an average size of $40 \mathrm{~nm}$. In the first method, the aqueous $\mathrm{AgNO}_{3}$ solution was treated with trisodium citrate under heating condition near boiling point. The TEM features clearly reveal that these particles are separated and are not uniform.

The mechanism of the reaction in this method could be expressed as follows:

$$
\begin{aligned}
& 4 \mathrm{Ag}^{+}+\mathrm{C}_{6} \mathrm{H}_{5} \mathrm{O}_{7} \mathrm{Na}_{3}+2 \mathrm{H}_{2} \mathrm{O} \\
& \quad \rightarrow 4 \mathrm{Ag}^{0}+\mathrm{C}_{6} \mathrm{H}_{5} \mathrm{O}_{7} \mathrm{H}_{3}+3 \mathrm{Na}^{+}+\mathrm{H}^{+}+\mathrm{O}_{2} \uparrow
\end{aligned}
$$

The colloidal gold nanoparticles formed by the process above remain in suspension by their mutual electrostatic repulsion generated and maintained by a net negative charge on their surface. One can understand that, since the silver nanoparticles possessed a negative charge due to the adsorbed citrate ions, a repulsive force worked along the particles and prevented aggregation. Therefore, the particles in the solution remain in a stable state without using other stabilizing agents.

In order to prepare the ultrafine AgNPs, in the second experiment, sodium citrate was replaced with sodium borohydride, which is a strong reducing agent. The aim was to improve the size distribution of the prepared silver nanoparticles. This method produced ultrasmall and well-controlled size of silver nanoparticles. By using sodium borohydride, the chemical reaction is the sodium borohydride reduction of silver nitrate: $\mathrm{AgNO}_{3}+\mathrm{NaBH}_{4} \rightarrow \mathrm{Ag}+{ }^{1 / 2} \mathrm{H}_{2}+{ }^{1 / 2} \mathrm{~B}_{2} \mathrm{H}_{6}+\mathrm{NaNO}_{3}$. The TEM micrograph (Figure 2a) shows spherical AgNPs deposited onto a carbon-coated copper grid, and the size histogram (Figure 2b) shows that the particles are in the range of 1 to $12 \mathrm{~nm}$ with an average diameter of $4 \mathrm{~nm}$.

This method produced ultrasmall silver particles. However, there must be enough borohydride to stabilize the particles as the reaction proceeds. However, later in the reaction, too much sodium borohydride increases the overall ionic strength, and aggregation will occur. Nanoparticles were kept in suspension by repulsive electrostatic forces between the particles, owing to adsorbed borohydride. The colloidal silver solution then turned darker yellow, violet and finally grayish due to the aggregation. We can speculate that this method produced very small silver particles, but the solution remained in suspension in a short time. After 1 week, the color of the solutions changed into gray because of the collapse of the nanoparticles. Our aim was to synthesize a more stable colloidal silver solution for further use.

As a consequence, nanoparticles in colloidal solution can be stabilized by adsorbed PVP. Figure 3 a provides a clear TEM micrograph of spherical Ag nanoparticles (PVP-AgNPs) showing the particles are mainly monodispersed with an average size of $21 \mathrm{~nm}$. The preparation was carried out using additional sodium citrate and PVP as stabilizers and sodium borohydride as reducing agent. The role of PVP was to stabilize the nanoparticles. In the solution, the PVP bounded to the surface of silver particles; the amount of PVP in the solution and its molecular weight both influenced the average size and PVPadsorbed surface of the silver nanoparticles. We suppose here that the mechanism of PVP-adsorbed silver particles consists of two steps. In the first step, the silver ions were reduced by sodium borohydride to silver atoms, and then, nearby silver atoms aggregated at close range. The aggregates were the primary nanoparticles. Finally, the primary nanoparticles coalesced with other nearby primary nanoparticles or interacted with PVP to form larger aggregates, 


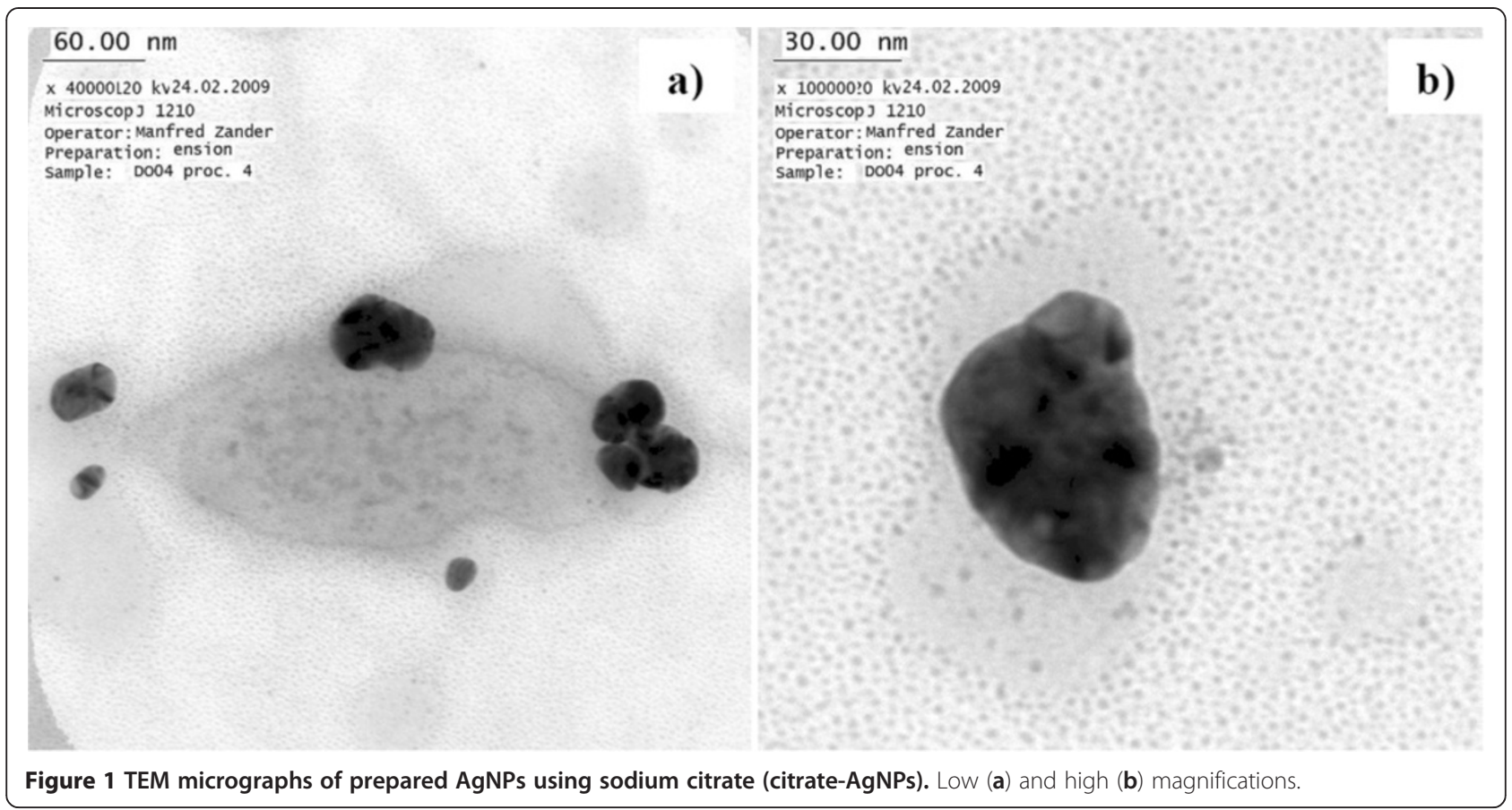

which were the secondary nanoparticles [8]. During this process, PVP was expected to inhibit the further aggregation of silver nanoparticles and to stabilize the dispersed silver nanoparticles in the solution [28]. Silver particles in this solution existed longer than those synthesized by using sodium citrate and sodium borohydride without using any stabilizing agent. It can be pointed out that the role of PVP is very important to keep those particles isolated and stable for months even at room temperature.

\section{Triangular Silver Nanoprisms}

Silver nanoprisms were synthesized via chemical reduction of silver nitrate. When the sodium citrate and hydrogen peroxide were added before $\mathrm{NaBH}_{4}$, the solution acquired first a yellow color, but after $1 \mathrm{~h}$, the color had changed to blue. This change was due to the change of surface plasmon resonance of metallic nanostructures. The morphology study of the triangular solution (Figure $4 \mathrm{a}-\mathrm{d}$ ) by transmission electron microscopy
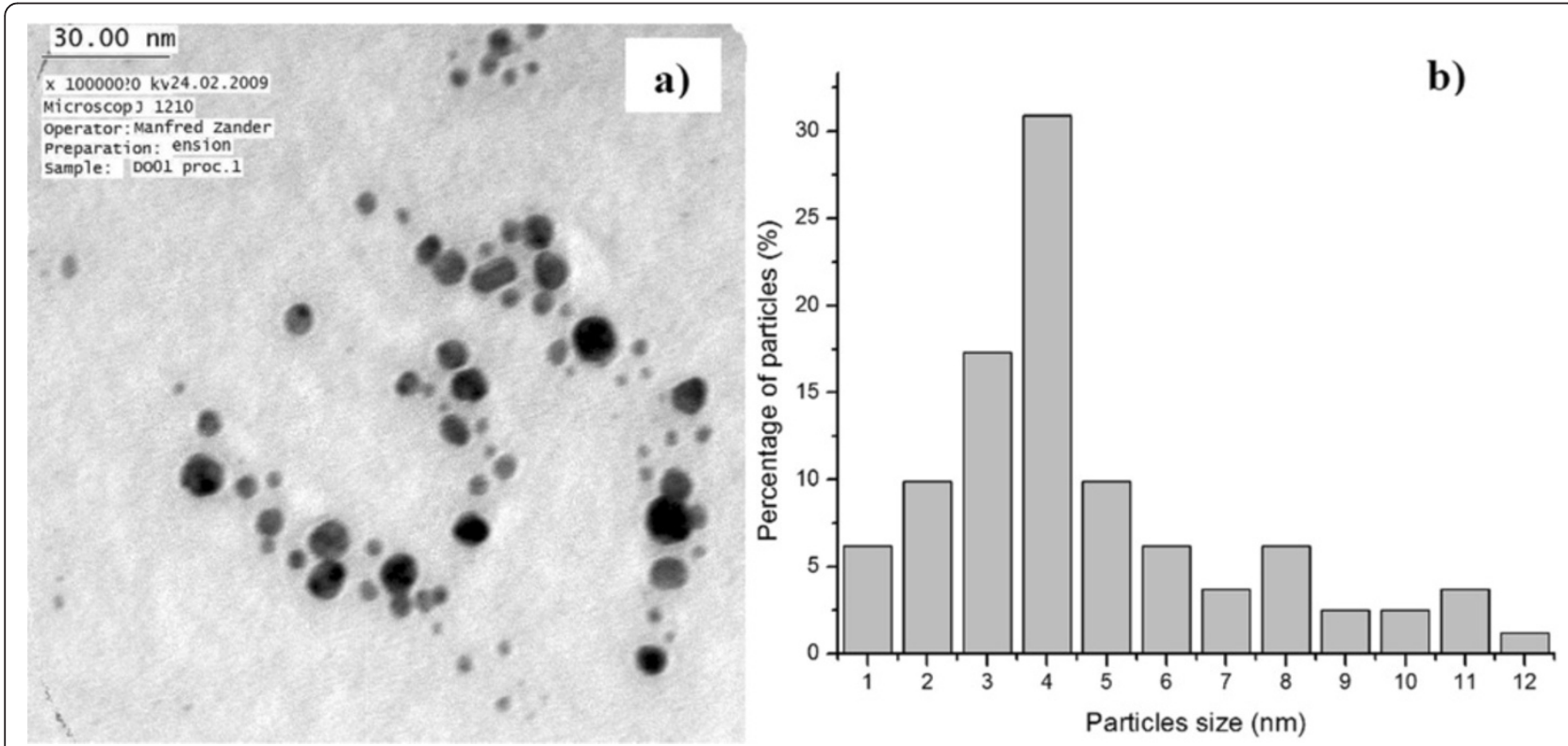

Figure 2 Ultrafine AgNPs prepared using the second method. (a) A TEM micrograph of silver nanoparticles dispersed on a copper grid prepared by $\mathrm{NaBH}_{4}$. (b) Histogram showing size distribution of borohydride-AgNPs. 

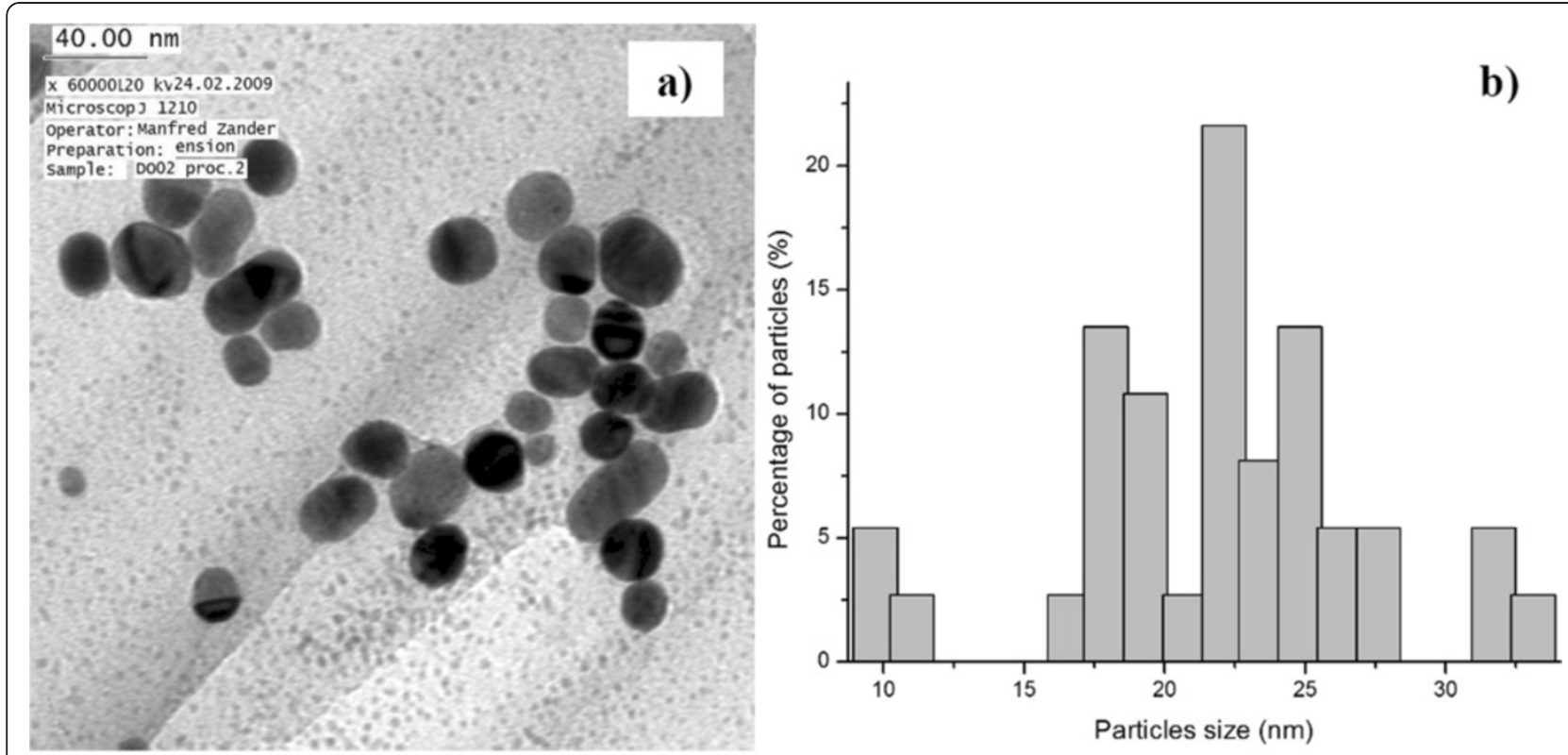

Figure 3 TEM micrograph of PVP-AgNPs. Dispersed on a cooper grid (a) and (b) histogram of size distribution.

to determine shape and size clearly reveals the presence of silver nanoprisms in a very broad size distribution ranging from 25 to $400 \mathrm{~nm}$. The triangular nanoprisms found in this study were very unimodal with sharp edges and sharp vertexes. However, when we prolonged the time of reaction, there appeared the large triangular silver nanoprisms (Figure 4c,d) in the solution.

Crystalline information of the edged triangular nanoprisms was obtained by particulate monolayer XRD measurement. The X-ray diffraction measurement (Figure 5) also indicates that the remarkably intensive diffraction of the characteristic peak at a $2 \theta$ value of 38.1 corresponds to the reflections of metallic silver particles crystallized in the face-centered cubic (fcc) structure with basal $\{111\}$ lattice planes.

A proposed reaction mechanism for the anisotropic transition from silver nanoparticles to nanoprisms in this approach could be explained by the use of a strong reductant (sodium borohydride) to induce the reduction of silver nitrate and formation of small spherical silver nanoparticles at room temperature when added immediately after sodium citrate, and using hydrogen peroxide to induce further oxidation of small particles into $\mathrm{Ag}^{+}$ ions and their transformation into nanoprisms at room temperature. Therefore, the presence of hydrogen peroxide in combination with sodium citrate, PVP and sodium borohydride is important for the synthesis of silver nanoprisms [27].

\section{UV-Vis spectrum analysis}

UV-vis spectroscopy is one of the most widely used techniques for structural characterization of silver nanoparticles. The adsorption spectra of the yellow silver solution (Figure 6a) prepared by sodium borohydride reduction in the presence of PVP shows the surface plasmon resonance at about $420 \mathrm{~nm}$, indicating the presence of spherical and roughly spherical Ag nanoparticles with an average size of $21 \mathrm{~nm}$ as confirmed by TEM photographs.

On the other hand, the UV-vis spectrum of the blue silver solution (Figure 6b) clearly reflects their anisotropic shape. The absorption spectrum showed a surface plasmon absorption band, which indicates the presence of fine triangular silver nanoparticles. Instead of a single peak at about $420 \mathrm{~nm}$ characteristic of spherical particles, two peaks appeared at 340 and $710 \mathrm{~nm}$. These bands in the region of 600 to $700 \mathrm{~nm}$, as well as one in approximately $340 \mathrm{~nm}$, correspond to the in-plane dipole plasmon and out-of-plane quadrupole resonance, suggesting the formation of silver nanoparticles with triangular nanoprism structures at the end of the reaction [27]. This interpretation of the UV-vis measurement agrees with the TEM measurement showing triangular nanoprisms and rough nanoprisms.

\section{Energy-dispersive X-ray spectroscopy analysis}

The elemental analysis of the silver nanoparticles was performed using the energy-dispersive X-ray spectroscope (EDX) equipped on the TEM. The EDX spectrum (Figure 7) shows that the prepared samples are pure silver (with clear Ag peaks) with no contaminated substances. Peaks corresponding to $\mathrm{C}$ and $\mathrm{Cu}$ are due to the elements of the carbon-coated copper grid in the TEM measurement. 


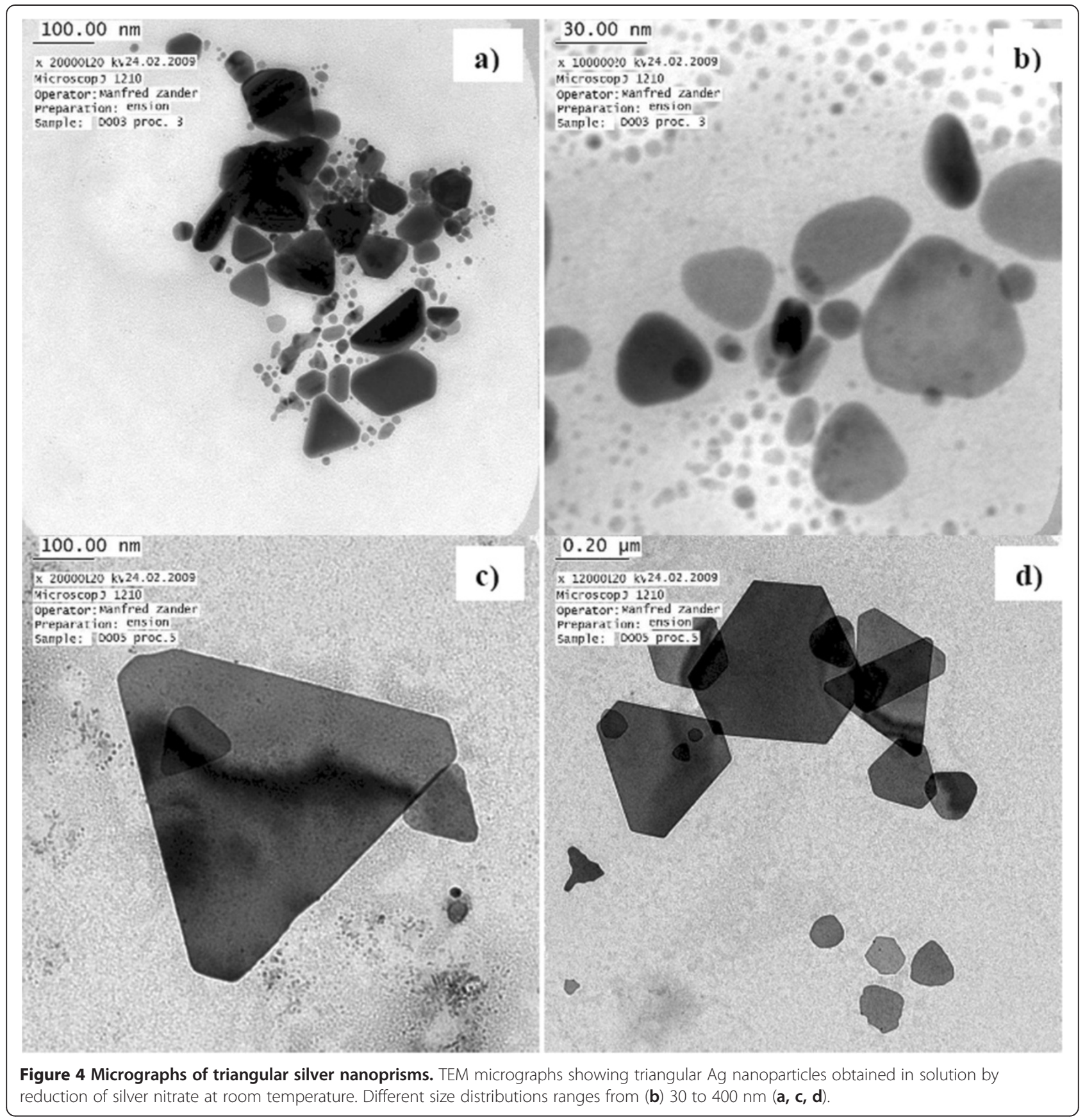

\section{Antimicrobial activity study}

Antibacterial test by zone of inhibition was carried out to qualitatively determine the level of inhibition by using differently prepared colloidal silver nanoparticles. Figure 8 shows the antibacterial zone of citrate-AgNPs (Figure 8a), PVP-AgNPs (Figure 8b) and borohydrideAgNPs (Figure 8c), respectively. As the bacteria grew to form a confluent lawn, the growth inhibition could be measured as the expansion of the clear zones surrounding the holes on the Petri dish.
Silver nanoparticles inhibited bacterial growth by the clear bacterial inhibition zone (a certain concentration of silver at $6 \mu \mathrm{g} / \mathrm{ml}$ ), which is correlated with the antibiotic activity of $\mathrm{Ag}$ nanoparticles (Table 1). Different sizes of spherical AgNPs were estimated in which ultrasmall borohydride-AgNPs (mean size $4 \mathrm{~nm}$ ) showed the strongest antibacterial activity, compared to larger spherical particles. The citrate-AgNPs with a broad size distribution (15 to $60 \mathrm{~nm}$ ) confirmed by TEM exhibited the lowest antibacterial activity. As a result, small size was 


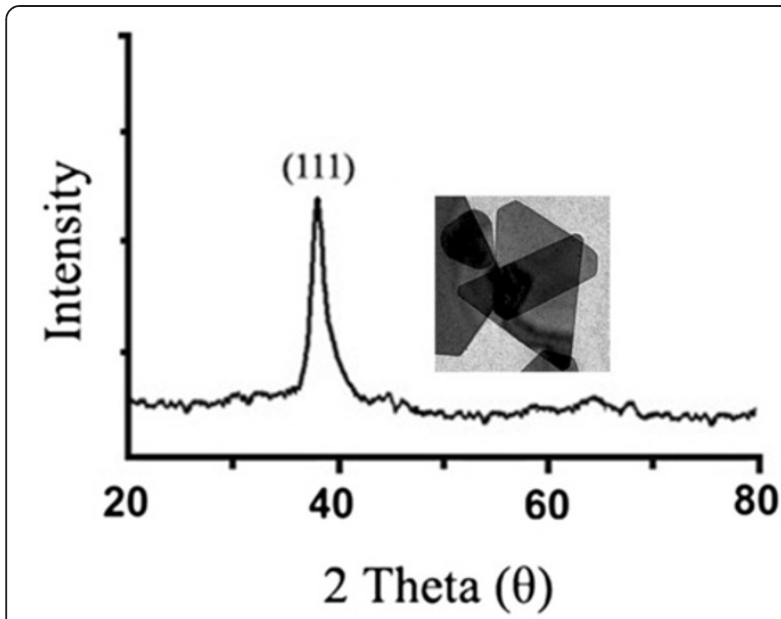

Figure 5 XRD pattern of triangular silver nanoprisms.

proven to have greater antibacterial properties against the bacteria. Ultrasmall size enables particles to penetrate easily into the cell wall and ultralarge surface area per mass where a large number of atoms are in immediate contact with the ambiance and readily available for reaction with the components of the cell.

This result confirmed the hypothesis and the work of Morones et al. [29]. In this study, we expected that the silver nanoparticles with differently morphological shapes would show a novel antibacterial activity in comparison to the conventionally spherical shapes.

By means of antibacterial zone expansion (at the same content of $4 \mu \mathrm{g} / \mathrm{ml}$ of silver), the silver solution containing triangular silver nanoprisms showed a greater inhibition activity against bacteria, compared with spherical nanoparticles (Figure 9). As the results show, the triangular silver nanoprisms have stronger antibacterial property than that of the spherical ones at the same concentration of silver and even at different sizes (4-nm borohydride-AgNPs and 25- to 400-nm triangular

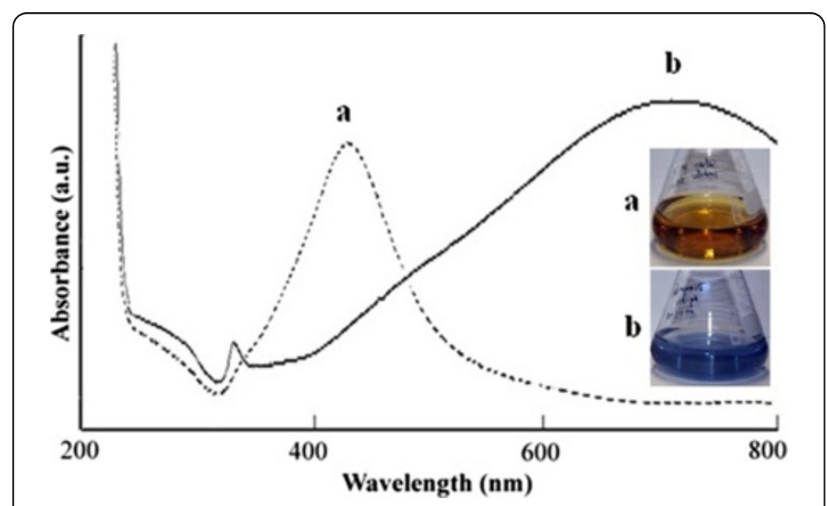

Figure 6 The UV-visible spectrum of (a) spherical PVP-AgNPs and (b) triangular nanoprisms.

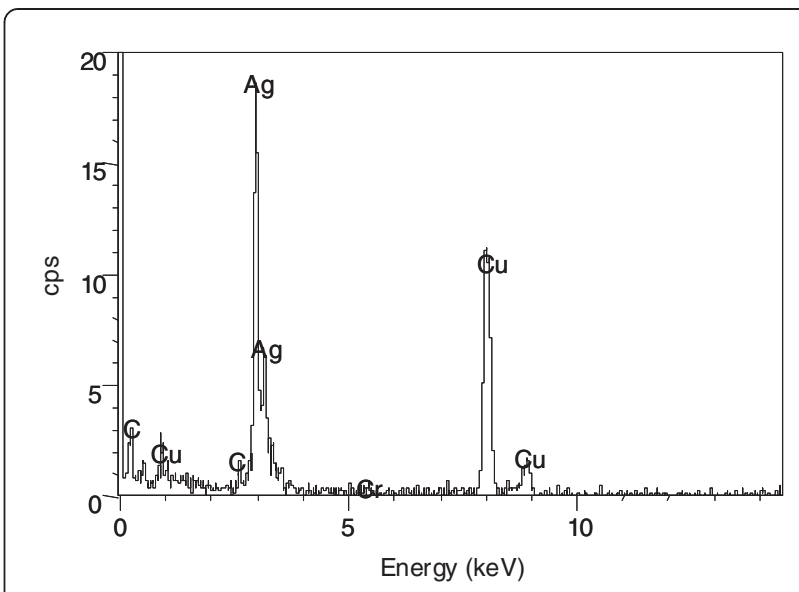

Figure 7 An EDX spectrum of a sample from a selected area.

nanoprisms). These results can be explained as demonstrated in the works by Pal et al. [25] and Morones et al. [29] where the authors concluded that the nanocrystals with $\{111\}$ basal plane has the strongest activity against the bacteria due to the high-atom-density $\{111\}$ facets. Thus, a high reactivity of triangular nanoplates in comparison to spheres, as found in this study, and partially silver spheres that contain fewer $\{111\}$ facets is expected. However, in this study, we used the triangular nanoprisms which contain sharp edges and vertexes, while in the study of Pal et al., the antibacterial activity of the truncated triangular particles was found. We suggest that these edges and vertexes influence the antibacterial activity; specifically, the vertex of triangular nanoprisms may contribute a very important role of harming bacterial cell wall in which the vertexes enable silver prisms to easily penetrate into the cell. In the previous study, Morones et al. demonstrated that the smaller size makes particles penetrate the cell easily, but in this study, we suggest a hypothesis that the geometrically triangular nanoprism having very sharp vertexes and sharp edges would be more toxic in damaging the bacterial cell.

\section{Conclusions}

A variety of chemical synthesis methods have been investigated for the preparation of different silver nanoparticle solutions. In each case, particular attention was paid to modification of the nanoparticle average size as well as the shape distribution. These properties were characterized by UV-vis spectroscopy, TEM, EDX and XRD. Different spherical silver nanoparticles were synthesized in a broad size distribution of 4, 21 and $40 \mathrm{~nm}$, respectively. Nanosized triangular prisms with sharp edges and vertexes were, on the other hand, prepared in water at room temperature in the presence of hydrogen peroxide with the size ranging from 25 to $400 \mathrm{~nm}$. The UV-vis spectrum features non-spherical 

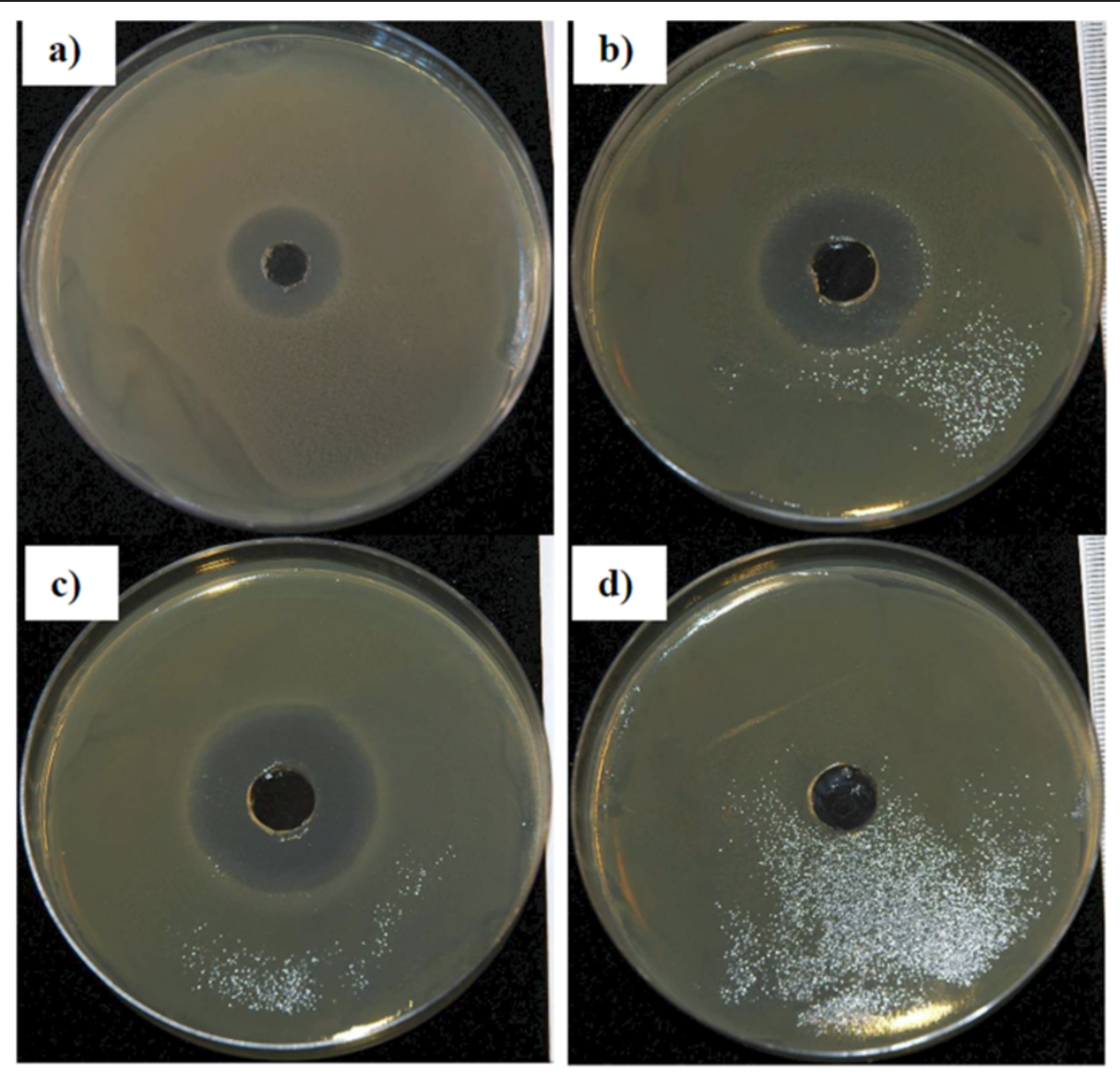

Figure 8 Petri dishes supplemented with $6 \mu \mathbf{g} / \mathbf{m l}$ pure silver with different spherical Ag nanoparticles. (a) Citrate-AgNPs, (b) PVP-AgNPS, (c) borohydride-AgNPs and (d) $\mathrm{H}_{2} \mathrm{O}$.

shape in different resonance frequencies at 340 and $710 \mathrm{~nm}$, while spherical silver nanoparticles show one plasmon peak at approximately $420 \mathrm{~nm}$.

Zone inhibition test of antibacterial activity showed that triangular silver nanoprisms have greater antibacterial activity compared with spherical silver nanoparticles due to their geometric structures and $\{111\}$ crystal planes, and small-size particles also confer greater antibacterial properties against the bacteria. The result revealed that the triangular nanoprisms with sharp edges and vertexes pose very high antibacterial properties, compared with spherical-shaped silver nanoparticles. As a result, synthesis and characterization of silver nanostructures with regard to novel morphology are of great interest in the fabrication of antibacterial materials.

Table 1 Antibacterial effect of different spherical silver nanoparticles

\begin{tabular}{llll}
\hline & Citrate-AgNPs & PVP-AgNPs & Borohydride-AgNPs \\
\hline $\begin{array}{l}\text { E. coli (extended } \\
\text { zone size) }\end{array}$ & $0.5 \mathrm{~cm}$ & $0.8 \mathrm{~cm}$ & $1.3 \mathrm{~cm}$ \\
\hline
\end{tabular}

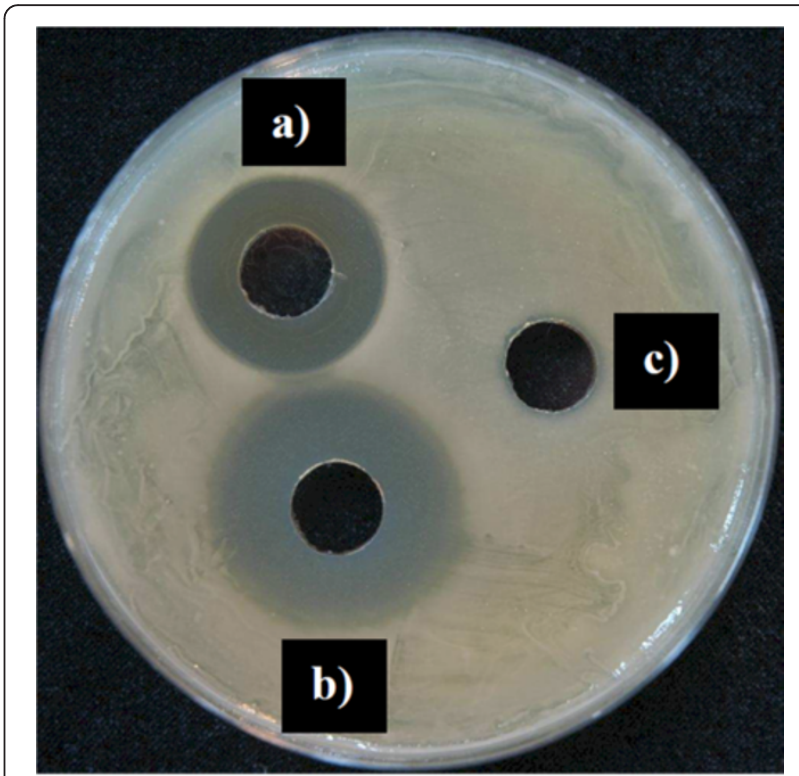

Figure 9 Extended inhibition zones. (a) Spherical silver nanoparticles (borohydride-AgNPs) and (b) silver nanoprisms and (c) $\mathrm{H}_{2} \mathrm{O}$. 


\section{Competing interests}

The authors declare that they have no competing interests.

\section{Acknowledgments}

Financial support from the project HSP "Molecular Biotechlogy" in the frame of DAAD funded by the German government is gratefully acknowledged.

\section{Author details}

${ }^{1}$ Institute of Material Science, Vietnam Academy of Science and Technology, 18 Hoang Quoc Viet Street, CauGiay District, Hanoi 010000, Vietnam. ${ }^{2}$ Institute of Biotechnology, Vietnam Academy of Science and Technology, 18 Hoang Quoc Viet Street, CauGiay District, Hanoi 010000, Vietnam. ${ }^{3}$ Institute of Geography and Geology, University of Greifswald, Makarenkostrasse 22, 17487 Greifswald, Germany.

\section{Authors' contributions}

PVD prepared the design of the study with experimental procedures and prepared the manuscript. JK supervised and conducted the progress of the study and also provided finance for the operation of this work. $\mathrm{CHH}$ and LTB inspired ideas and design throughout the study. All authors read and approved the final manuscript.

\section{Authors' information}

PVD graduated from the University of Engineering and Technology, Vietnam National University in engineering physics and has a Master degree in nanomaterials and devices. He used two years working at the Institute of Biotechnology before joining a training course in Greifswald University, Germany. Currently, he is working at the Institute of Materials Science, Vietnam Academy of Science and Technology (VAST) on both nanomaterials and nanobiotechnology aspects.

$\mathrm{CHH}$, an associate professor, earned his PhD degree in Germany and is currently the president of the Plant Cell Biotechnology Department, Institute of Biotechnology, VAST. He is very interested in nanotechnology and particularly bionanotechnology. He is very dedicated to the students and has guided many students in biotechnology.

LTB, a professor, is a senior scientist and a former president of the Institute of Biotechnology, VAST. He had a long time working in Germany as a very brilliant scientist. He is very dedicated to science and is known as a very famous scientist in plant cell biotechnology in Vietnam.

JK is a professor and a German scientist in the Institute of Geography and Geology, University of Greifswald. He is very brilliant in TEM and electron microscopy techniques. He conducted many projects in cooperation with the Vietnamese and German government.

Received: 30 March 2012 Accepted: 21 June 2012

Published: 21 June 2012

\section{References}

1. Warheit, D.B., Borm, P.J., Hennes, C., Lademann, J.: Testing strategies to establish the safety of nanomaterials: conclusions of an ECETOC workshop. Inhal Toxicol 19, 631-643 (2007)

2. Chen, X., Schluesener, H.J.: Nanosilver: a nanoproduct in medical application. Toxicol Lett 176, 1-12 (2008)

3. Mallick, K., Witcomb, M.J., Scurrell, M.S.: Polymer stabilized silver nanoparticles: a photochemical synthesis route. J Mater Sci 39, 4459-4463 (2004)

4. Dolgaev, S.I., Simakin, A.V., Voronov, V.V., Shafeev, G.A., Bozon-Verduraz, F.: Nanoparticles produced by laser ablation of solids in liquid environment. App. Surf. Sci 186, 546-551 (2002)

5. Li, D., Chen, S., Zhao, S., Hou, X., Ma, H., Yang, X.: A study of phase transfer processes of Ag nanoparticles. App. Surf. Sci. 200, 62-67 (2002)

6. Husein, M., Rodil, E., Vera, J.H.: Formation of silver bromide precipitate of nanoparticles in a single microemulsion utilizing the surfactant counterion. J Colloid Interface Sci 273, 426-434 (2004)

7. Salkar, R.A., Jeevanandam, P., Aruna, S.T., Koltypin, Y., Gedanken, A.: The sonochemical preparation of amorphous silver nanoparticles. J. Mater. Chem. 9, 1333-1335 (1999)

8. Shin, H., Yang, H., Kim, S., Lee, M.: Mechanism of growth of colloidal silver nanoparticles stabilized by polyvinyl pyrrolidone in $\gamma$-irradiated silver nitrate solution. J Colloid Interface Sci 274, 89-94 (2004)
9. Chaudhari, P.R., Masurkar, S.A., Shidore, V.B., Kamble, S.P.: Antimicrobial activity of extracellularly synthesized silver nanoparticles using lactobacillus species obtained from VIZYLAC capsule. J. App. Pharmaceutical. Sci 02, 2529 (2012)

10. Masurkar, S.A., Chaudhari, P.R., Shidore, V.B., Kamble, S.P.: Rapid biosynthesis of silver nanoparticles using Cymbopogan citratus (lemongrass) and its antimicrobial activity. Nano-Micro. Lett. 3, 189-194 (2011)

11. Chan, Y.S., Mashitah, M.D.: Instantaneous biosynthesis of silver nanoparticles by selected macro fungi. Australian J. Basic. App. Sci 6(1), 86-88 (2012)

12. Dawy, M., Rifaat, H.M., Moustafa, S.A., Mousa, H.A.: Physicochemical studies on nano silver particles preparated by different techniques. Australian J. Basic. App. Sci 6(3), 257-262 (2012)

13. Prabhu, N., Divya, T.R., Yamuna, G.: Synthesis of silver phyto nanoparticles and their antibacterial efficacy. Digest. J. Nanomater. Biostruct 5, 185-189 (2010)

14. Khan, Z., Al-Thabaiti, S.A., Obaid, A.Y., Al-Youbi, A.O.: Preparation and characterization of silver nanoparticles by chemical reduction method. Colloid. Surf. B: biointerfaces 82, 513-517 (2011)

15. Sadeghi, B., Jamali, M., Kia, S., Amininia, A., Ghafari, S.: Synthesis and characterization of silver nanoparticles for antibacterial activity. Int. J. Nano. Dim 1(2), 119-124 (2010)

16. Hussain, J.I., Kumar, S., Hashmi, A.A., Khan, Z.: Silver nanoparticles: preparation, characterization, and kinetics. Adv. Mat. Lett 2(3), 188-194 (2011)

17. Galdiero, S., Falanga, A., Vitiello, M., Cantisani, M., Marra, V., Galdiero, M.: Silver nanoparticles as potential antiviral agents. Molecules 16, 8894-8918 (2011)

18. Shrivastava, S., Bera, T., Roy, A., Singh, G., Ramachandrarao, P., Dash, D.: Characterization of enhanced antibacterial effects of novel silver nanoparticles. Nanotechnology 18, 225103 (2007)

19. Mpenyana-Monyatsi, L., Mthombeni, N.H., Onyango, M.S., Momba, M.N.B. Cost-effective filter materials coated with silver nanoparticles for the removal of pathogenic bacteria in groundwater. Int J Environ Res Public Health 9, 244-271 (2012)

20. Tang, B., Wang, J., Xu, S., Afrin, T., Xu, W., Sun, L., Wang, X.: Application of anisotropic silver nanoparticles: multifunctionalizationof wool fabric. J Colloid Interface Sci 356, 513-518 (2011)

21. Chaloupka, K., Malam, Y., Seifalian, A.M.: Nanosilver as a new generation of nanoproduct in biomedical applications. Trends Biotechnol 28, 580-588 (2010)

22. Afreen, R.V., Ranganath, E.: Synthesis of monodispersed silver nanoparticles by Rhizopus Stolonifer and its antibacterial activity against MDR strains of Pseudomonas Aeruginosa from burnt patients. Int. J. Environ. Sci 1(7), 15821592 (2011)

23. Savithramma, N., Rao, M.L., Rukmini, K., Suvarnalatha, P.D.: Antimicrobial activity of silver nanoparticles synthesized by using medicinal plants. Int. J. Chem.Tech. Research. (USA) 3, 1394-1402 (2011)

24. Mirzajani, F., Ghassempour, A., Aliahmadi, A., Esmaeili, M.A.: Antibacterial effect of silver nanoparticles on Staphylococcus aureus. Research. Microbiol $162,542-549(2011)$

25. Pal, S., Tak, Y., Song, J.: Does the antibacterial activity of silver nanoparticles depend on the shape of the nanoparticle? A study of the gram-negative bacterium Escherichia coli. App. Environ. Microbiol 73(6), 1712-1720 (2007)

26. Fang, J., Zhong, C., Mu, R.: The study of deposited silver particulate films by simple method for efficient SERS. Chemical Physics Letters 401, 271-275 (2005)

27. Torres, V., Popa, M., Crespo, D., Moreno, J.M.: Silver nanoprism coatings on optical glass substrates. Microelectronic Engineering 84, 1665-1668 (2007)

28. Pastoriza-Santos, I., Liz-Marzan, L.M.: Formation of PVP-protected metal nanoparticles in DMF. Langmuir. 18, 2888-2894 (2002)

29. Morones, J.R., Elechiguerra, J.L., Camacho, A., Holt, K., Kouri, J.B., Ramirez, J.T., Yacaman, M.J.: The bactericidal effect of silver nanoparticles. Nanotechnology 16, 2346-2353 (2005)

doi:10.1186/2228-5326-2-9

Cite this article as: Van Dong et al.: Chemical synthesis and antibacterial activity of novel-shaped silver nanoparticles. International Nano Letters 2012 2:9. 